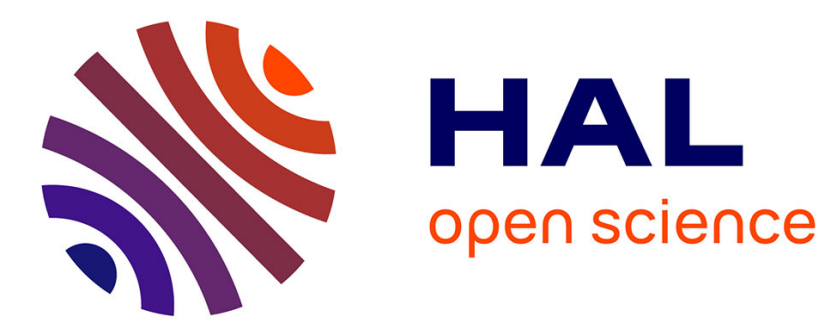

\title{
Postural configuration affects the perception of earth-based space during pitch tilt
}

\author{
Aurore Bourrelly, J. -L. Vercher, L. Bringoux
}

\section{To cite this version:}

Aurore Bourrelly, J. -L. Vercher, L. Bringoux. Postural configuration affects the perception of earth-based space during pitch tilt. Acta Psychologica, 2011, 138 (1), pp.119-125. 10.1016/j.actpsy.2011.05.014 . hal-01436026

\section{HAL Id: hal-01436026 \\ https://hal.science/hal-01436026}

Submitted on 2 May 2018

HAL is a multi-disciplinary open access archive for the deposit and dissemination of scientific research documents, whether they are published or not. The documents may come from teaching and research institutions in France or abroad, or from public or private research centers.
L'archive ouverte pluridisciplinaire HAL, est destinée au dépôt et à la diffusion de documents scientifiques de niveau recherche, publiés ou non, émanant des établissements d'enseignement et de recherche français ou étrangers, des laboratoires publics ou privés. 


\title{
Postural configuration affects the perception of earth-based space during pitch tilt
}

\author{
A. Bourrelly *, J.-L. Vercher, L. Bringoux * \\ UMR CNRS 6233 “Institut des Sciences du Mouvement Etienne-Jules Marey," CNRS and Aix-Marseille Université, France
}

\section{A R T I C L E I N F O}

Article history:

Received 22 October 2010

Received in revised form 22 May 2011

Accepted 24 May 2011

Available online 12 June 2011

PsycINFO codes:

2340 Cognitive Processes

2320 Sensory Perception

2221 Sensory \& Motor Testing

4090 Transportation

\section{Keywords:}

Spatial perception

Postural orientation

Reference frame

Geocentric

Egocentric

\begin{abstract}
A B S T R A C T
This study investigates the relative contribution of body parts in the elaboration of a whole-body egocentric attraction phenomenon previously observed during earth-based judgments. This was addressed through a particular earth-based task requiring estimating the possibility of passing under a projected line, imagining a forward horizontal displacement. Different postural configurations were tested, involving whole-body tilt, trunk tilt alone or head tilt alone. Two legs positions relative to the trunk were manipulated. Results showed systematic deviations of the subjective "passability" toward the tilt, linearly related to the tilt magnitude. For each postural configuration, the egocentric influence appeared to be highly dependent on the position of trunk and head axes, whereas the legs position appeared not relevant. When compared to the whole-body tilt condition, tilting the trunk alone consistently reduced the amount of the deviation toward the tilt, whereas tilting the head alone consistently increased it. Our results suggest that several specific effects from multiple body parts can account for the global deviation of the estimates observed during whole-body tilt. Most importantly, we support that the relative contribution of the body segments could mainly depend on a reweighting process, probably based on the reliability of sensory information available for a particular postural set.
\end{abstract}

(c) 2011 Elsevier B.V. All rights reserved.

\section{Introduction}

The visual horizon, defined as the plane normal to gravity crossing eye level (Stopper \& Cohen, 1989) has been found critically involved in the perception of earth-based space. Several studies have shown that estimating distance (Ooi, Wu, \& He, 2001), elevation (Matin \& Li, 1995), and the possibility of passing under high obstacles (Bringoux, Robic, Gauthier, \& Vercher, 2008) relies on this reference. Most of the time, the visual horizon is fully accessible, or can be derived from the contextual lines of a structured visual environment if not directly available (Wu, He, \& Ooi, 2005). However, in absence of vision (during night or foggy day), the spatial judgments mentioned above must rely on an implicit horizontal reference named the subjective visual horizon (SVH).

Numerous studies indicated that the visual horizon was generally perceived $-2^{\circ}$ below the physical reference, when measured in darkness for erect subjects (e.g., McDougall, 1903; Howard, 1986). They also showed that the SVH could be influenced by numerous environmental factors such as the orientation of the visual scene (Matin \& Li, 1995), and the gravitational flow field (Tribukait \&

\footnotetext{
* Corresponding authors at: UMR CNRS 6233, CNRS and Aix-Marseille Université, 163, avenue de Luminy, CP 910, 13288 Marseille, France. Tel.: + 3349117 22 62; fax: + 33491172252 .

E-mail addresses: aurore.bourrelly@hotmail.fr (A. Bourrelly), lionel.bringoux@univmed.fr (L. Bringoux).
}

Einken, 2005). Interestingly, body orientation was also found to significantly influence the SVH (about $20 \%$ of body tilt magnitude in the direction of pitch body tilt, and in a range from $\pm 20^{\circ}$ ), stressing the importance of taking into account the whole-body position in space when making earth-based spatial judgements (Bourrelly, Bringoux, \& Vercher, 2009; Bourrelly, Vercher, \& Bringoux, 2010; Bringoux, Tamura, Faldon, Gresty, \& Bronstein, 2004; Bringoux et al., 2008). Nevertheless, the origin of this whole-body tilt influence remains unclear.

The main effect of body tilt upon SVH was initially interpreted as a decreased sensitivity of the vestibular system during tilt, leading to a diminished sensation of tilt (Lechner-Steinleitner, 1978; Shöne, 1964; Young, 1984). Similar interpretation could be given considering the phenomenon of somatosensory adaptation observed after prolonged tilt (Higashiyama \& Koga, 1998; Wade, 1970). However, this hypothesis was inconsistent with other studies which found no relationship between the estimated body orientation and the perception of earth-based references (Mast \& Jarchow, 1996, Mittelstaedt, 1996; Trousselard, Barraud, Nougier, Raphel, \& Cian, 2004). Another interpretation of the relationship observed between body orientation and SVH has been recently suggested in terms of egocentric attraction induced by body tilt (Bringoux et al., 2004, 2008).

Here, we addressed the issue of the possible link between this latter interpretation and the idiotropic vector hypothesis previously formulated by Mittelstaedt (1983). This hypothesis considers that the 
longitudinal Z-body axis could serve as a strong reference in estimating some relevant earth-based directions such as the subjective visual vertical and the SVH. According to the previous explanation, geocentric judgements would be attracted towards the whole Z-body axis, regardless of the perception of tilt (Carriot, DiZio, \& Nougier, 2008). This whole-body attraction has been reported more or less important, however, depending on the dimension of body tilt (roll vs. pitch; Ebenholtz, 1970) or the direction to be estimated (vertical vs. horizontal; Betts \& Curthoys, 1998; Carriot et al., 2008; Lejeune, Thouvarecq, Anderson, Caston, \& Jouen, 2009). Alternatively, the $Z$-trunk axis and the $Z$-head axis were also shown to constitute relevant egocentric references influencing the perception of earthbased directions (Guerraz, Poquin, Luyat, \& Ohlmann, 1998; Wade, 1969, 1970; Wetzig \& Baumgarten, 1990). For instance, Wetzig and Baumgarten (1990) and Guerraz et al. (1998) showed a specific effect of roll head tilt on judgments of verticality which was smaller than during whole-body tilt, supporting the assumption that multiple body parts could be taken into account in the elaboration of a whole-body egocentric attraction. Moreover, Guerraz et al. (1998) suggested that the single effects relative to the tilt of the $Z$-trunk axis and the $Z$-head axis could be additively combined into a main egocentric effect when the head and trunk were tilted together. However, this hypothesis of additivity between independent body parts has not been systematically accepted. Ito and Gresty (1996) supported the theory of a dynamic combination of multiple body parts such as legs, trunk and head position in the elaboration of a main egocentric effect. Specifically, they suggested that the weight attributed to each single body part could evolve during the task such as the more the subjects are tilted backward, the more the weight attributed to the trunk-leg axis is important. In addition, the egocentric attraction during tilt was found greater for erect subjects (with the head to trunk-leg axis in alignment) than for seated subjects. These results strongly suggested that the postural configuration in space could affect a large number of spatial tasks relied on earth-based directions.

The aim of the present study was to determine the origin of the egocentric attraction previously observed on earth-based judgments during whole-body tilt. The question was addressed by testing the influence of postural configuration on a particular earth-based task which requires to estimate the possibility of passing under high obstacles (Bourrelly et al., 2009; Bringoux et al., 2008). Indeed, it has been previously demonstrated that the perceived ability of passing under obstacles in otherwise darkness is related to the perceived earth-based horizon at eye level, acting as a reference for height judgements (Bringoux et al., 2008; Marcilly \& Luyat, 2008). In Experiment 1, we examined the contribution of head, trunk and leg positions in the elaboration of the whole-body egocentric attraction previously observed in the judgements of "passability" under a projected horizontal line. In Experiment 2, we focused on the influence of active head orientation in the same task to further investigate the contribution of somatosensory and vestibular inputs in the elaboration of the main egocentric attraction effect.

\section{General methods}

\subsection{Apparatus}

In the subsequent experiments reported here, subjects were seated on a padded tilting chair allowing body rotations in pitch within a range from $+20^{\circ}$ backward to $-20^{\circ}$ forward with accelerations above the vestibular threshold for rotation perception. Prior to any condition, subjects were restrained by means of a shoulder harness with their head strapped on a head-and-chinrest so that the naso-occipital axis was orthogonal to the direction of gravity when the chair was vertically oriented. Eye level was positioned so that the trans-ocular axis coincided to the axis of rotation of the chair. Consequently, eye level was kept at the same height relative to the floor reference $(1.34 \mathrm{~m})$ whatever the body tilt magnitude. Depending on the experimental condition, the head could be kept either vertical while the trunk was tilted, aligned with the trunk during whole-body tilts, or tilted alone while the trunk was kept vertical. The feet were strapped onto an adjustable foot-rest which permitted to reach specific legs positions (flexed vs. extended) relative to the body.

Subjects were placed in front of a flat vertical screen $2 \mathrm{~m}$ height $\times 2.5$ wide at a distance of $2.28 \mathrm{~m}$ from the eyes. A laser pointer located behind the screen projected a thin horizontal beam on a tilting mirror. The luminous line was reflected on the screen. The elevation of the projected line was adjustable in height by means of a galvanometer (Scanner Control CCX 100) which allowed the rotation of the mirror in pitch. The resulting luminous horizontal line was $2 \mathrm{~m}$ long and $0.01 \mathrm{~m}$ thick and adjustable in height with a precision of $0.01 \mathrm{~m}$. Subjects held in both hands the digital response push buttons for judgment settings. Galvanometer control and response recordings were performed by the ADwin-Pro system (Keithley@) piloted via our in-house software (Docometre). All the judgments were performed in a dark room to avoid external visual cues (Fig. 1).

\subsection{General procedure}

Nine angles of tilt were manipulated in the present study. For each body orientation, 10 line elevations were randomly presented. Subjects were asked to answer the following question: "Do you think you could pass under the line in the present body orientation, imagining a forward horizontal displacement of your body?". To make sure that the subjects clearly understood the task, sketches were presented, illustrating a forward horizontal displacement (always normal to gravity) and passable or impassable obstacles for different postural configurations. Subjects were first positioned at the desired body angle relative to gravity in complete darkness. The chair was rotated at a constant velocity during $11 \mathrm{~s}$, with a period of initial acceleration and final deceleration of $2 \mathrm{~s}\left(0.4^{\circ} \mathrm{s}^{-1}\right.$ and $0.2^{\circ} \mathrm{s}^{-2}$ for $\pm 5^{\circ}$ tilt, $0.8^{\circ} \mathrm{s}^{-1}$ and $0.4^{\circ} \mathrm{s}^{-2}$ for $\pm 10^{\circ}$ tilt, $1.2^{\circ} \mathrm{s}^{-1}$ and $0.6^{\circ} \mathrm{s}^{-2}$ for $\pm 15^{\circ}$ tilt, $1.6^{\circ} \mathrm{s}^{-1}$ and $0.8^{\circ} \mathrm{s}^{-2}$ for $\pm 20^{\circ}$ tilt). This was followed by $15 \mathrm{~s}$ of rest. This specific duration was chosen as a compromise between the weakest vestibular resting discharge allowing to consider post-rotational effects as negligible and limited somatosensory adaptation due to the subsequent period of static tilt (Benson, 1990; Goldberg \& Fernandez, 1977). Stationary subjects were then asked to open their eyes and to gaze at the horizontal line which appeared on the screen during $4 \mathrm{~s}$. At the end of the visual presentation, the line was switch off and subjects were asked to respond about the possibility of passing under the line, via a forcedchoice judgment by means of two hand-held buttons. Judgment settings were recorded via the ADwin-Pro system (Keithley@) piloted via our in-house software (Docometre). At the end of the sequence (i.e., a successive presentation of 10 line elevations in a same body orientation), the chair was brought back to the vertical and the room lights were turned on for $10 \mathrm{~s}$ before a new sequence was launched. The instructions were frequently repeated to keep subjects alert and concentrated on the task throughout the experiment. Subjects were neither informed about the number and height of line elevations.

\subsection{Data processing}

Judgment settings were first converted into binary values. A score of 1 was attributed when the subjects estimated they could pass under the line, that is, when the line elevation was perceived higher than the minimal height for passing under. Conversely, a score of 0 was attributed when the subjects estimated they could not pass under the line. A subsequent "Probit" model, using a nonlinear regression analysis for dichotomic variables, was used to determine the probability $p$ that subjects estimated at 50\% that they 


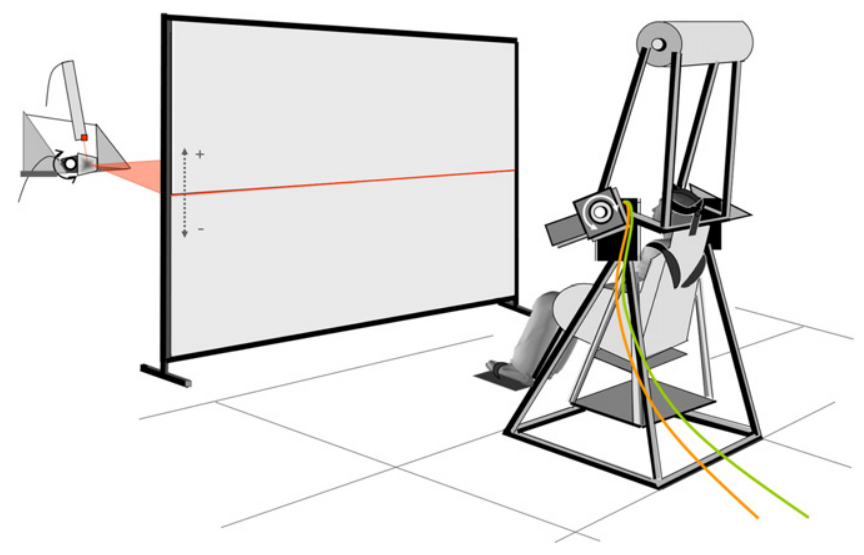

Fig. 1. Illustration of the experimental set-up. The motorized chair rotating around the subjects' trans-ocular axis could be rotated from $+20^{\circ}$ backward to $-20^{\circ}$ forward. Depending on the experimental condition, subjects head could be either kept vertical while the trunk was tilted, aligned with the trunk during whole-body tilts, or tilted alone while the trunk was kept vertical. The feet were strapped onto an adjustable footrest which permitted to reach flexed or extended legs positions relative to the body. The luminous horizontal line was projected from a laser beam at different elevations on the screen. Subjects had to fix the line on the screen and estimate whether they could pass under, imagining a forward horizontal displacement.

could pass under the line. Line elevations obtained at $p=0.5$ via the psychometric function defined judgements of subjective "passability", that is, estimates of the minimal height relative to eye level required for passing under obstacles (see Bourrelly et al., 2009). The slope of the tangent at the inflection point of the Probit curve gave an indication about the discrimination sensitivity of the so-called subjective "passability" relative to the chosen increments. The sharper the slope, the higher the discrimination sensitivity. Analyses of variance (ANOVA) with repeated measures were performed on the slopes of the Probit function to ensure there was no difference in the discrimination sensitivity whatever the experimental condition. The estimates of subjective "passability" initially referred to eyelevel for convenience, were subsequently referred to the top of the head (the highest physical point of the head from the horizontal floor reference) measured for each subject in each body orientation. Hence, the data were expressed as a vertical elevation (in $\mathrm{cm}$ ) relative to the top of the head in order to define a true level of "passability" Repeated measures ANOVAs were performed on the data to test the presence of a specific egocentric influence in each postural configuration at the different angles of tilt. Linear regression lines were then applied to the estimates of subjective "passability" for each subject in each postural configuration to characterize the nature of the egocentric influence. Differences between postural configurations were tested by comparing the slopes of the regression lines obtained for each subject. NewmanKeuls post-hoc tests were used to characterize the effects.

\section{Experiment 1}

The purpose of the Experiment 1 was to investigate the contribution of head, trunk and legs position in the elaboration of the whole-body egocentric attraction previously reported in the judgments of "passability" under high obstacles.

\subsection{Methods}

\subsubsection{Subjects}

Eight subjects (four males and four females; mean age 23.4 \pm 4.2 year) with normal or corrected to normal vision (by lens correction) gave informed consent to participate in the study, in compliance with the ethical committee which regulates human experimentation in France. They had no previous history of vestibular and neurological symptoms. All were naive as to the hypothesis under study.

\subsubsection{Experimental conditions}

Four postural configurations were tested in the present experiment (Fig. 2). The effects of the whole-body orientation, trunk orientation, and legs position were investigated. The experimental conditions were named as following: B (whole-body tilt with extended legs), B-Lflex (whole-body tilt with flexed legs), T (trunk tilt alone with the extended legs), and T-Lflex (trunk tilt alone with flexed legs). For each condition, the head was secured by means of a head-and-chinrest, either mounted on the tilting display (so that head-and-trunk was tilted as a whole during whole-body rotation) or fixed in space (so that the trunk was tilted alone with the head remaining fixed). The feet were secured to an adjustable footrest. The position of the legs, flexed or extended, was determined so that the axis from the malleolus to the eye axis respectively reaches an angle of
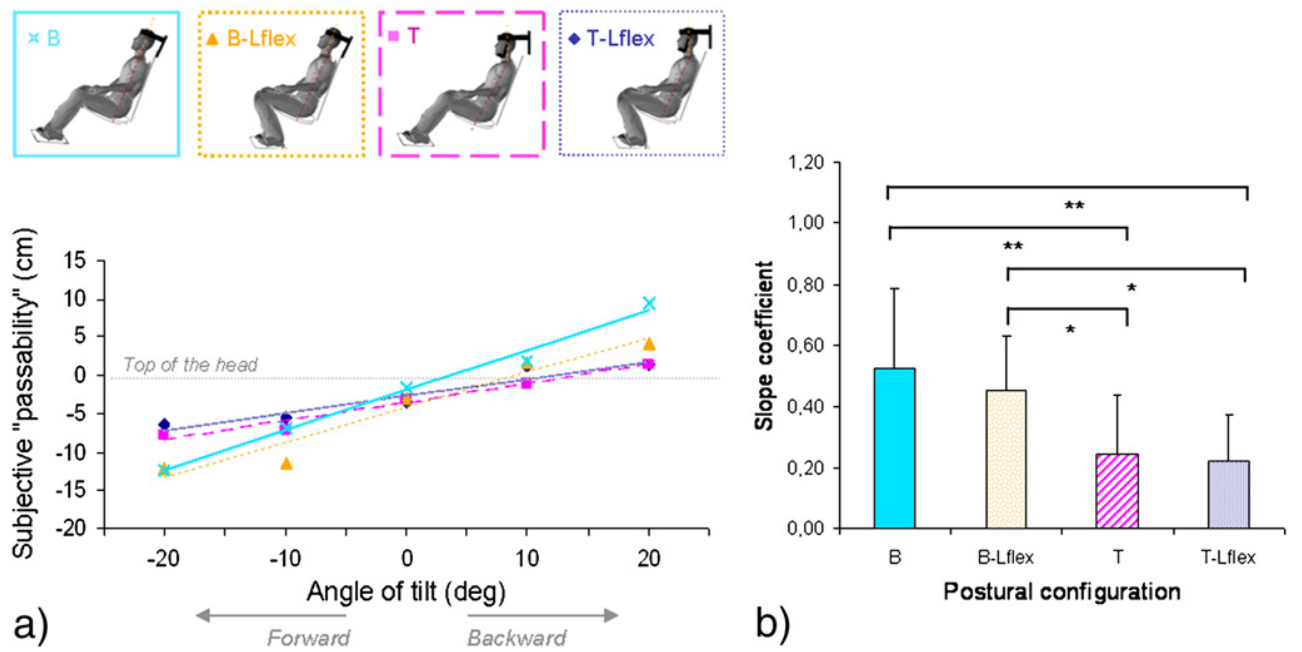

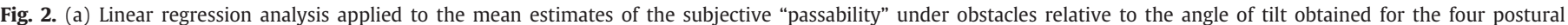

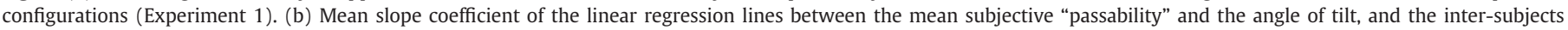

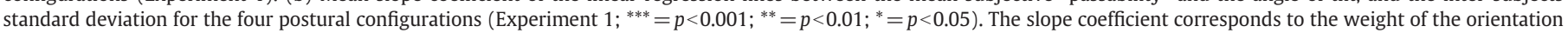
influence. 
$35^{\circ}$ or $45^{\circ}$ relative to gravity. The order of the tested different body positions was initially randomized and then counterbalanced for half of the subjects (i.e., strict inverse order in the presentation of the postural configurations for two sub-groups).

\subsubsection{Procedure}

Five angles of tilt relative to gravity (i.e. whole-body tilt or trunk tilt alone; $0^{\circ} ;-10^{\circ},-20^{\circ}$ forward; and $+10^{\circ},+20^{\circ}$ backward) were manipulated in the present experiment. For each body orientation, 10 line elevations $(+5,+10,+15,+20,+25 \mathrm{~cm}$ upward elevations; and $-5,-10,-15,-20,-25 \mathrm{~cm}$ downward elevations $\mathrm{cm}$ from eye level; i.e., respectively, $\pm 1.3^{\circ}, \pm 2.5^{\circ}, \pm 3.8^{\circ}, \pm 5.0^{\circ}, \pm 6.3^{\circ}$ elevations from eye level) were pseudo-randomly presented. To avoid any order effects, the order of presentation was strictly counterbalanced for half of the subjects. The total number of judgments was $400(4 \times 5 \times 10)$ for a total session duration of $90 \mathrm{~min}$.

\subsection{Results and discussion}

A non-linear regression analysis (Probit function) was performed to determine the subjective "passability" for each subject in each experimental condition (see "General methods"). A one-way ANOVA with repeated measures on the slopes of the Probit function curve was performed for each body orientation. The analysis showed no significant difference in the discrimination sensitivity of the subjective "passability" whatever the body orientation $(F(3,12)=0.79$, $p=0.52$ ). A 4 postural configurations $\times 5$ angles of tilt ANOVA with repeated measures on each factor was then conducted on the estimates of subjective "passability" Results showed a significant difference between the manipulated angles of tilt on the subjective "passability" $(F(4,28)=30, p<0.001)$ but no significant main difference between the postural configurations $(F(3,21)=0.65, p=0.59)$. Nevertheless, the interaction between the angle of tilt and the postural configuration was highly significant $(F(12,84)=3.59$; $p<0.001)$. This suggested that the tilt effect was clearly dependant on the postural configuration.

In order to further characterize the influence of body orientation upon the judgements, linear regression analyses were applied to the estimates of subjective "passability" obtained for each subject in each experimental condition (Fig. 2-a). Results, summarized in Table 1, showed a significant linear influence of body orientation on the estimated possibility of passing under high obstacles in the four postural configurations. Specifically, the level of subjective "passability" was systematically deviated in the direction of tilt, that is, the more the subjects were tilted backward, the more they felt possible to pass under a given obstacle. Equations of the regression lines performed on the mean subjective estimates for the four experimental conditions are expressed in the following terms $Y=a \theta-b$, where the slope coefficient " $a$ " corresponds to the weight of the orientation influence, " $\theta$ " to angle of tilt, " $Y$ " to the subjective "passability" and " $b$ " to a negative offset characterizing the general lowering of the subjective estimates relative to the true level of "passability"

In order to compare the magnitude of the "tilt influence" (i.e., the deviation of the subjective "passability" in the direction of tilt) between the different postural configurations, a one-way ANOVA with

Table 1

Results of the linear regression analysis between the mean subjective "passability" and the angle of tilt (Experiment 1).

\begin{tabular}{lllll}
\hline Experimental conditions & $\begin{array}{l}\text { Equation of the } \\
\text { regression lines }\end{array}$ & $R 2$ & $p$ \\
\hline B & (Whole-body) & $Y=0.53 \theta-1.90$ & $R 2=0.99$ & $p<.001$ \\
B-Lflex & (Whole-body - legs flexed) & $Y=0.46 \theta-4.18$ & $R 2=0.94$ & $p<.05$ \\
T & (Trunk alone) & $Y=0.24 \theta-3.55$ & $R 2=0.97$ & $p<.01$ \\
T-Lflex & (Trunk alone - legs flexed) & $Y=0.22 \theta-2.59$ & $R 2=0.92$ & $p<.01$ \\
\hline
\end{tabular}

repeated measures was conducted on the slope coefficients derived from the individual regression lines for each postural configuration (Fig. 2-b). Results showed significant differences between postural configurations $(F(3,21)=7.99, p<0.001)$. Specifically, post-hoc analyses (Newman-Keuls test) showed a significant influence of the head position in space (i.e., significant differences between $B$ and $\mathrm{T}$ condition; $p<0.01$ ) but no significant differences in the legs position relative to the trunk (i.e., no differences between the $\mathrm{B}$ and B-Lflex condition, and between the $\mathrm{T}$ and T-Lflex condition).

The egocentric effect for the B and B-Lflex condition was about 46 and $53 \%$ of the tilt magnitude, respectively. Noteworthily, the weight obtained for the whole-body orientation influence is fully comparable with the one previously described in the literature for similar judgments of "passability" under obstacles (45\%; Bourrelly et al., 2009). Interestingly, fixing the head in space appears to notably reduce the weight of the egocentric attraction observed on perceptual judgments of "passability" under obstacles. This can be explained by the fact that the head- $Z$ axis, which is kept aligned with gravity, constitutes a stabilizing reference for earth-based judgments (Pozzo, Papaxanthis, Stapley, \& Berthoz, 1998). Nevertheless, regarding the weight of the egocentric attraction obtained for each experimental condition, the results showed that the trunk orientation influence (between 22 and $24 \%$ of the tilt magnitude) can account for almost half of the whole-body egocentric attraction in both legs positions. However, no direct conclusion can be done about the relative contribution of head orientation in the elaboration of the wholebody egocentric attraction. This is precisely the aim of Experiment 2 to question this point.

\section{Experiment 2}

The purpose of the Experiment 2 was to further investigate the influence of head orientation in the egocentric attraction effect previously reported in literature. Active head orientation, rather than passive head orientation, was manipulated with the assumption that active head movements could improve the subjective "passability" under obstacles (Fouque, Bardy, Stoffregen, \& Bootsma, 1999; Viviani, 1990). Particularly, active head orientation could contribute to diminish the effect of egocentric attraction from the head tilt by providing additional information of the head position relative to gravity (Gooey, Bradfield, Talbot, Morgan, \& Proske, 2000; Luyat, Gentaz, Regia Corte, \& Guerraz, 2001). Specific effects of head and trunk orientation were evaluated separately and compared to the whole-body egocentric effect obtained in a range of $\pm 20^{\circ}$ pitch tilts.

\subsection{Methods}

\subsubsection{Subjects}

Eight new subjects (three males and five females; mean age $25.25 \pm$ 2.9 year) with normal or corrected to normal vision (by lens correction) participated in this second experiment. They had no previous history of vestibular and neurological symptoms. None of them took part in the previous experiment to avoid any prior knowledge relative to the hypotheses under study.

\subsubsection{Experimental conditions}

Three postural configurations were tested to address the influence of the whole-body tilt (B), the trunk tilt alone $(\mathrm{T})$, and the head tilt alone $(\mathrm{H})$ on the subjective "passability" under obstacles (Fig. 3). To allow comparisons with the Experiment 1, the B and T conditions were the same as previously described. In the $\mathrm{H}$ condition, the head was tilted alone relative to gravity while the chair was kept vertically oriented. 

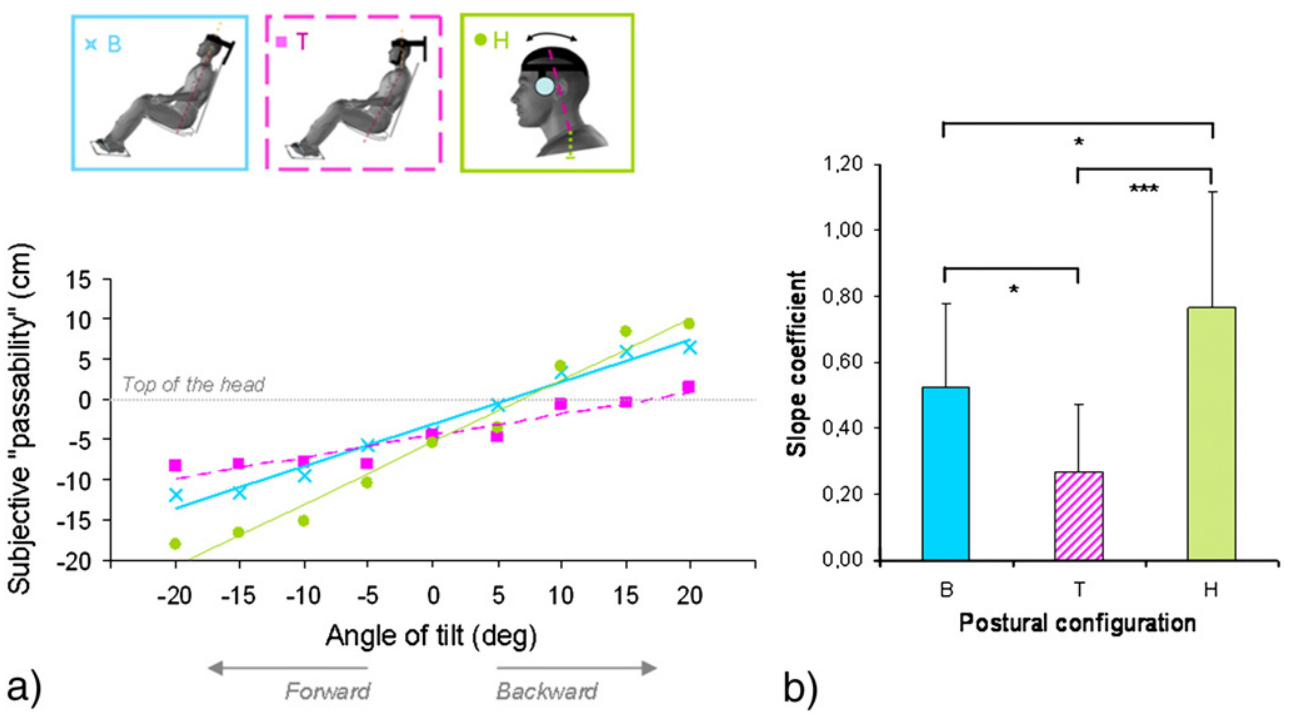

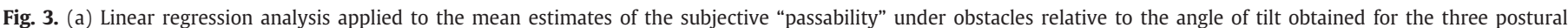

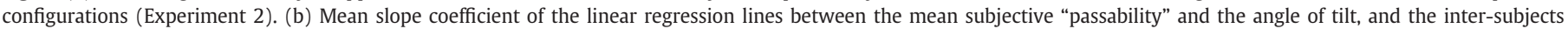

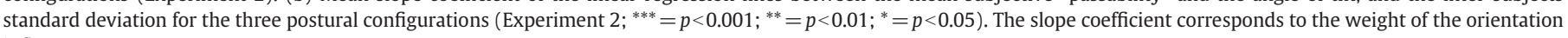
influence.

\subsubsection{Procedure}

Subjects' head was first positioned at the desired angle. The orientation was controlled on line by the experimenter by means of an inclinometer (AccuStar ${ }^{\circledR}$ ). Eye level was positioned at a constant height ( $1.34 \mathrm{~m}$ relative to the floor reference, and 2.28 relative to the screen) by adjusting the chair in height and depth. Subjects were asked to keep the head orientation still until the end of the trial. If the signal of head position changed during the judgments by more than 1 degree, the trial was canceled and presented again later in the session.

Nine angles of tilt relative to gravity $\left(0^{\circ} ;-5^{\circ},-10^{\circ},-15^{\circ},-20^{\circ}\right.$ forward; and $+5^{\circ},+10^{\circ},+15^{\circ},+20^{\circ}$ backward) were manipulated in the present experiment. For each body or head orientation, twelve visual stimuli $(0 ;+5,+10,+15,+20,+25,+35 \mathrm{~cm}$ upward elevations; and $-5,-10,-15,-20,-25 \mathrm{~cm}$ downward elevations $\mathrm{cm}$ from eye level; i.e., respectively, $0, \pm 1.3^{\circ}, \pm 2.5^{\circ}, \pm 3.8^{\circ}, \pm 5.0^{\circ}$, $\pm 6.3^{\circ}$ and $+7.5^{\circ}$ elevations from eye level) were presented to the subjects in a pseudo-randomized order. For a given body or head orientation, each visual stimulus was repeated 3 times in a pseudorandomized order. This order was strictly counterbalanced for half of the subjects. Finally, the total number of judgments was 324 $(3 \times 9 \times 12)$ for a total session duration of $60 \mathrm{~min}$. Except for the previous points, the experimental set-up and procedure were the same as in the Experiment 1.

\subsection{Results and discussion}

As for Experiment 1, no significant difference was found in the discrimination sensitivity of the Probit function whatever the body orientation magnitude $(F(2,16)=0.15, p=0.86)$. A 3 postural configurations $\times 9$ angles of tilt ANOVA with repeated measures on each factor was then conducted on the mean estimates of subjective "passability" Results showed a significant difference between the manipulated angles of tilt $(F(8,56)=31.65, p<0.0001)$ but no significant main difference between the postural configurations $(F(2,14)=0.23, p=0.8)$. Nevertheless, the interaction between the angle of tilt and the postural configuration was highly significant $(F(16,112)=4.94 ; p<0.0001)$. Here again, this clearly suggested that the tilt effect appeared dependant on the postural configuration.

Linear regression analyses were performed on the subjective "passability" obtained for each subject in each experimental condition (Fig. 3-a). Results confirmed a linear effect of the whole-body tilt (B) and the trunk tilt alone (T) on estimating the possibility of passing under high obstacles, as observed in Experiment 1. In addition, results showed a linear effect of head tilt alone $(\mathrm{H})$ on the perceptual estimates. The equations of the regression lines calculated on the mean subjective estimates were summarized in Table 2.

In order to compare the linear influences between the different postural configurations, a one-way ANOVA with repeated measures was conducted on the slope coefficients derived from the regression lines for each postural configuration. Results, summarized in Fig. 3-b showed significant differences between the three postural configurations $(F(2,14)=12.77, p<0.001)$. Post-hoc analyses (Newman-Keuls test) are reported in Fig. 3-b.

Regarding the slope coefficients of the regression lines obtained for each postural configuration, the "whole-body tilt" (B) condition and the "trunk tilt alone" (T) condition show comparable weights in both Experiments 1 and 2. As for Experiment 1, the weight of the egocentric attraction seems to be half of the whole-body egocentric attraction when the head is fixed in space. Conversely, tilting the head alone induced a greater egocentric attraction than when the whole-body is tilted. In this latter $(\mathrm{H})$ condition, one must acknowledge the presence of supplementary motor information resulting from the active support of the head (i.e., efference copy). While further investigations need to be conducted to disambiguate the role of "active" vs. "passive" proprioception, our data strongly suggest a relevant implication of the combined vestibular and neck proprioceptive information in the observed egocentric attraction.

\section{General discussion}

The aim of the present study was to determine the origin of the egocentric attraction previously observed on earth-based judgments.

Table 2

Results of the linear regression analysis between the mean subjective "passability" and the angle of tilt (Experiment 2).

\begin{tabular}{lllll}
\hline $\begin{array}{l}\text { Experimental } \\
\text { conditions }\end{array}$ & Equation of the regression lines & $R 2$ & $p$ \\
\hline $\mathrm{B}$ & (Whole-body) & $Y=0.52 \theta-3.10$ & $R 2=0.98$ & $p<.001$ \\
$\mathrm{~T}$ & (Trunk alone) & $Y=0.27 \theta-4.60$ & $R 2=0.90$ & $p<.001$ \\
$\mathrm{H}$ & (Head alone) & $Y=0.77 \theta-5.34$ & $R 2=0.97$ & $p<.001$ \\
\hline
\end{tabular}


To address this question, we investigated the contribution of head, trunk and legs position, in the elaboration of the whole-body egocentric attraction previously reported on estimating the possibility of passing under high obstacles during pitch body tilt. The main finding of this study was that the estimated possibility of passing under high obstacles depends on both the magnitude of tilt and the postural configuration in space. Whole-body, trunk and head orientations were found to exert a significant linear effect on perceptual judgments in a range of $\pm 20^{\circ}$ of tilt. In other words, systematic deviations of the subjective "passability" were observed toward the tilt, and were proportional to the tilt magnitude. For each postural configuration, the egocentric influence appeared also to be highly dependent on the position of trunk and head axis in space. For instance, when compared to the whole-body tilt condition, tilting the trunk alone was found to consistently reduce the amount of the deviation toward the tilt, whereas tilting the head alone was found to consistently increase the deviation toward the tilt.

Previous studies suggested that specific egocentric effects from several body parts could merge together to influence earth-based judgments (Bourrelly et al., 2009; Guerraz et al., 1998; Ito \& Gresty, 1996; Mittelstaedt, 1983). However, the way this combination would be achieved remains unclear. Two main interpretations can be advanced from the literature. A first hypothesis suggested that trunk and head specific effects may originate from independent egocentric influences, which could additively combine to yield a main egocentric attraction on earth-based judgments. In line with this hypothesis, Bourrelly et al. (2009) showed that, in a range of $\pm 10^{\circ}$ of tilt, gaze and body orientation participated each with a constant weight in the elaboration of the main egocentric attraction by a simple summation process. This hypothesis of additivity was also supported by the work of Guerraz et al. (1998) and Wetzig and Baumgarten (1990). Both studies suggested that specific head roll influence could account for the whole-body egocentric attraction in a main part, the remaining part being due to the trunk influence. Our data showed that the weight corresponding to the effect of the trunk orientation alone is half of that obtained for the whole-body orientation. If such an additive process is at work in the elaboration of the main egocentric attraction, the effect of head orientation should account for the other half in the elaboration of the main whole-body egocentric effect. However, the sum of the single effects observed in our study when the head and the trunk are tilted independently gives rise to a larger egocentric influence than when the whole-body is tilted. This suggests that the egocentric attraction may stem from a more complicated process than a simple summation of single and independent effects related to the orientation of different body parts. In other words, our results did not support the idea of an "absolute" and invariant weight attributed to each body segment, irrespective of the postural configuration.

Hence, a second hypothesis may be advanced to explain how the body segments may combine to yield a main egocentric influence on earth-based judgments. The main assumption is that a reweighting process may occur between the different body parts and their respective egocentric influence, depending on the reliability of sensory cues available for a given postural configuration. By reweighting process, we name the interaction between several agents (e.g., sensory inputs, body parts, reference frames) whose influence (i.e., weight) may combine and evolve over time. By allocating a higher weight to reliable cues and a lower weight to unreliable ones, the central nervous system may optimize sensory integration and resolve sensory ambiguities about space representation for a given task (Ernst \& Banks, 2002; Ernst \& Bülthoff, 2004; Mars, Vercher, \& Popov, 2005). For instance, Mars et al. (2005) demonstrated that the respective weight attributed to vestibular and somatosensory cues were inversed, depending on whether the observers had to judge their self-orientation in space or objects orientation relative to gravity. In our study, the difference in sensory reliability may be related for a part to the nature of somatosensory and motor information involved in both tasks, that is information from trunk graviceptors, pressure cues from the skin, neck proprioceptive information or even efference copy during active tilt. For instance, trunk tilt alone induces neck proprioceptive changes, but no vestibular changes, which tends to confirm that vestibular cues are more reliable than somatosensory cues for the assessment of earth-based judgments when the head is vertically oriented (Wade, 1970). Conversely, a decreased vestibular reliability may occur during whole-body tilt (Schöne, 1964; Bringoux et al., 2004), hence explaining the increasing influence of tilt upon earthbased judgements. Furthermore, tilting the head alone induces neck proprioceptive and vestibular changes, as well as efferent information issued from active motor involvement (see 4.2 Results and discussion). Additional information from neck proprioception during head tilt alone, may it be actively maintained, could then provide ambiguous signals about whether the head is moving relative to the trunk or the trunk is moving relative to the head. In this condition, when available sensory cues are modified during tilt and may express different postural configurations, we hypothesized that the central nervous system may cautiously select the head orientation as a main reference for verticality during earth-based judgments. This point is supported by previous works indicating that the head constitutes a stabilized platform for numerous spatial tasks (Berthoz, 1997; Pozzo et al., 1998). Finally, one may summarize the latter interpretation by considering that the egocentric weight attributed to the $Z$-head axis is increased in case of head-and-trunk orientation dissociation.

This strongly challenges the assumption that active head movement could reduce the amount of errors in subjective "passability" by providing additional information about head position relative to gravity (Luyat et al., 2001). Previously, Bringoux et al. (2004) made a similar observation regarding the effect of active arm lifting on SVH judgements. Although arm lifting was supposed to provide additional information about gravity, the authors found that the SVH became more dependent on the whole-body tilt when judgements were performed through active arm movements. These observations stressed once again that estimating limb or body orientation in space and judging the location of earth-based references are likely based on different perceptual processes (Bronstein, 1999).

\section{Conclusion}

The present study demonstrates that estimating the subjective "passability" under high obstacles depends not only on the wholebody tilt magnitude, but also on the postural configuration in space. Head and trunk tilts were found to mainly attract the subjective "passability" toward their direction. Our results suggest that head and trunk influence could be reweighted to yield a main egocentric attraction, depending on the postural configuration. Special care should be addressed to the orientation of the head, as its egocentric weight may drastically increase when the head axis is not aligned with the trunk axis. The origin of such a reweighting process may probably stem from the reliability of the sensory information available for a particular postural set. Further investigations about the effect of passive vs. active head orientation alone may help to better understand the influence of motor involvement upon the wholebody egocentric attraction during static pitch tilt. In parallel, regarding the findings of Bringoux et al. (2009) who showed a dynamic evolution of the subjective vertical settings toward a tilted visual background, further experiments should be done to investigate how specific egocentric effects could evolve and merge over time. Finally, these findings could be of value in aeronautics where pilots, seated under different postural configurations depending on the type of aircraft, must achieve earth-based judgments in absence of a structured visual background, such as during night or foggy day. 


\section{Acknowledgements}

Aurore Bourrelly was supported by a grant from DGA-CNRS (No. 2007-746). The authors are grateful to F. Buloup, A. Donneaud and C. Goulon for technical expertise.

\section{References}

Benson, A. J. (1990). Sensory functions and limitations of the vestibular system. In R. Warren, \& A. H. Wertheim (Eds.), Perception and control of self-motion (pp. 145-170). Hillsdale: Lawrence Erlbaum Associates.

Berthoz, A. (1997). Le sens du mouvement. Paris: Odile Jacob.

Betts, G. A., \& Curthoys, I. S. (1998). Visually perceived vertical and visually perceived horizontal are not orthogonal. Vision Research, 38, 1989-1999.

Bourrelly, A., Bringoux, L., \& Vercher, J. L. (2009). Influence of gaze elevation on estimating the possibility of passing under high obstacles during body tilt Experimental Brain Research, 193, 19-28.

Bourrelly, A., Vercher, J. L., \& Bringoux, L. (2010). Pitch body orientation influences the perception of self-motion direction induced by optic flow. Neuroscience Letters, 482 , 193-197.

Bringoux, L., Bourdin, C., Lepecq, J. C., Sandor, P., Pergandi, J. M., \& Mestre, D. (2009) Interaction between reference frames during subjective vertical estimates in a tilted immersive virtual environment. Perception, 38, 1053-1071.

Bringoux, L., Robic, G., Gauthier, G. M., \& Vercher, J. L. (2008). Judging beforehand the possibility of passing under obstacles without motion: The influence of egocentric and geocentric frames of reference. Experimental Brain Research, 185, 673-680.

Bringoux, L., Tamura, K., Faldon, M., Gresty, M. A., \& Bronstein, A. M. (2004). Influence of whole-body pitch tilt and kinesthetic cues on the perceived gravity-referenced eye level. Experimental Brain Research, 155, 385-392.

Bronstein, A. M. (1999). The interaction of otolith and proprioceptive information in the perception of verticality. The effects of labyrinthine and CNS disease. Annals of the New York Academy of Sciences, 871, 324-333.

Carriot, J., DiZio, P., \& Nougier, V. (2008). Vertical frames of reference and control of body orientation. Neurophysiologie Clinique, 38, 423-437.

Ebenholtz, S. M. (1970). Perception of the vertical with body tilt in the median plane Journal of Experimental Psychology, 83, 1-6.

Ernst, M. O., \& Banks, M. S. (2002). Humans integrate visual and haptic information in a statistically optimal fashion. Nature, 415, 429-433.

Ernst, M. O., \& Bülthoff, H. (2004). Merging the senses into a robust percept. Trends in Cognitive Sciences, 8, 162-169.

Fouque, F., Bardy, B. G., Stoffregen, T. A., \& Bootsma, R. J. (1999). Action and intermodal information influence the perception of orientation. Ecological Psychology, 11, 1-43.

Goldberg, J. M., \& Fernandez, C. (1977). Conduction times and background discharge of vestibular afferents. Brain Research, 122, 545-550.

Gooey, K., Bradfield, O., Talbot, J., Morgan, D. L., \& Proske, U. (2000). Effects of body orientation, load vibration on sensing position and movement at the human elbow joint. Experimental Brain Research, 133, 340-348.

Guerraz, M., Poquin, D., Luyat, M., \& Ohlmann, T. (1998). Head orientation involvement in assessment of the subjective vertical during whole body tilt. Perceptual and Motor Skills, 87, 643-648.

Higashiyama, A., \& Koga, K. (1998). Apparent body tilt and postural aftereffect. Perception E' Psychophysics, 60, 331-347.
Howard, I. P. (1986). The perception of posture, self-motion, and the visual vertical. In K. Boff, L. Kaufman, \& J. Thomas (Eds.), Handbook of perception and human performance (pp. 1-62). New York: Willey.

Ito, Y., \& Gresty, M. A. (1996). Subjective postural orientation and visual vertical during slow pitch tilt for the seated human subject. Aviation, Space, and Environmental Medicine, 68, 3-12.

Lechner-Steinleitner, S. (1978). Interaction of labyrinthine and somatoreceptor inputs as determinants of the subjective vertical. Psychological Research, 40, 65-76.

Lejeune, L., Thouvarecq, R., Anderson, D. J., Caston, J., \& Jouen, F. (2009). Kinaesthetic and visual perceptions of orientations. Perception, 38, 988-1001.

Luyat, M., Gentaz, E., Regia Corte, T., \& Guerraz, M. (2001). Reference frames and haptic perception of orientation: Body and head tilt effects on the oblique effect. Perception \& Psychophysics, 63, 540-554.

MacDougall, R. (1903). The subjective horizon. Psychological Review, 4, 145-166.

Marcilly, R., \& Luyat, M. (2008). The role of eye height in judgment of an affordance of passage under a barrier. Current Psychology Letters, 24, 12-24.

Mars, F., Vercher, J. L., \& Popov, K. (2005). Dissociation between subjective vertical and subjective body orientation elicited by galvanic vestibular stimulation. Brain Research Bulletin, 65, 77-86.

Mast, F., \& Jarchow, T. (1996). Perceived body position and the visual horizontal. Brain Research Bulletin, 40, 393-398.

Matin, L., \& Li, W. (1995) Multimodal basis for egocentric spatial localization and orientation. Journal of Vestibular Research, 5, 499-518.

Mittelstaedt, H. (1983). A new solution to the problem of the subjective vertical. Naturwissenschaften, 70, 272-281.

Mittelstaedt, H. (1996). Somatic graviception. Biological Psychology, 42, 53-74.

Ooi, T. L., Wu, B., \& He, Z. J. (2001). Distance determined by the angular declination below the horizon. Nature, 414, 197-200.

Pozzo, T., Papaxanthis, C., Stapley, P., \& Berthoz, A. (1998). The sensorimotor and cognitive integration of gravity. Brain Research Reviews, 28, 92-101.

Shöne, H. (1964). On the role of gravity in human spatial orientation. Aerospace Medicine, 35, 764-772.

Stoper, A. E., \& Cohen, M. M. (1989). Effect of structured visual environments on apparent eye level. Perception \& Psychophysics, 46, 469-475.

Tribukait, A., \& Einken, O. (2005). Perception of the head transversal plane and subjective horizontal during gondola centrifugation. Perception E Psychophysics, 67, 369-382.

Trousselard, M., Barraud, P. A., Nougier, V., Raphel, C., \& Cian, C. (2004). Contribution of tactile and interoceptive cues to the perception of the direction of gravity. Cogn. Brain Res., 20, 355-362.

Viviani, P. (1990). Motor-perceptual interactions: The evolution of an idea. In M. Piattelli Palmarini (Ed.), Cognitive science in Europe: Issues and trends. Golem. (pp. 11-39).

Wade, N. J. (1969). Visual orientation as a function of head tilt. Percept. Motor. Skills., 29, 573-574.

Wade, N. J. (1970). Effect of prolonged tilt on visual orientation. Q. J. Exp. Psycol., 22, 423-439.

Wetzig, J., \& Baumgarten, R. J. (1990). Influence of neck receptor stimulation on eye rotation and on the subjective vertical: Experiment on the tilt table, under water, and weightlessness. In W. G. Berthoz, \& P. P. Vidal (Eds.), The head-neck sensorimotor system (pp. 198-200). Chichester: Wiley.

Wu, J., He, Z. J., \& Ooi, T. L. (2005). Visually perceived eye level and horizontal midline of the body trunk influenced by optic flow. Perception, 34, 1045-1060

Young, L. R. (1984). Perception of the body in space: Mechanisms. In I. Smith (Ed.), The nervous system. Handbook of Physiology. (pp. 1023-1066) New York: Academic Press. 